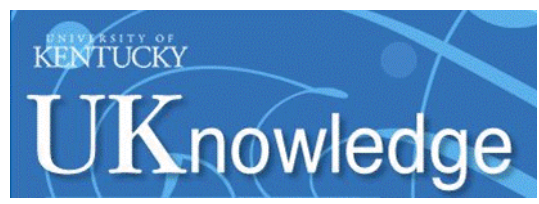

University of Kentucky

UKnowledge

\title{
Fluctuations in Astronomical Masers
}

Moshe Elitzur

University of Kentucky, moshe@pa.uky.edu

Follow this and additional works at: https://uknowledge.uky.edu/physastron_facpub

Part of the Astrophysics and Astronomy Commons, and the Physics Commons

Right click to open a feedback form in a new tab to let us know how this document benefits you.

\section{Repository Citation}

Elitzur, Moshe, "Fluctuations in Astronomical Masers" (1991). Physics and Astronomy Faculty Publications. 231.

https://uknowledge.uky.edu/physastron_facpub/231

This Article is brought to you for free and open access by the Physics and Astronomy at UKnowledge. It has been accepted for inclusion in Physics and Astronomy Faculty Publications by an authorized administrator of UKnowledge. For more information, please contact UKnowledge@lsv.uky.edu. 


\section{Fluctuations in Astronomical Masers}

Digital Object Identifier (DOI)

http://dx.doi.org/10.1086/185973

Notes/Citation Information

Published in The Astrophysical Journal Letters, v. 370, no. 1, p. L45-L48.

(C)1991. The American Astronomical Society. All rights reserved.

The copyright holder has granted permission for posting the article here. 
The Astrophysical Journal, 370:L45-L48, 1991 March 20

(C) 1991. The American Astronomical Society. All rights reserved. Printed in U.S.A.

\title{
FLUCTUATIONS IN ASTRONOMICAL MASERS
}

\author{
MOSHE ElitzUR \\ Department of Physics and Astronomy, University of Kentucky, Lexington, KY 40506 \\ Received 1990 October 19; accepted 1990 December 18
}

\begin{abstract}
The radiation of astronomical masers fluctuates on the time scale $\Gamma^{-1}$, where $\Gamma$ is the levels' loss rate. These intensity fluctuations reflect fluctuations of the level populations around their mean, steady state values over the length scale $\lambda_{c}=c / \Gamma$. In saturated masers, the intensity fluctuations are dominated by passage through the unsaturated core. The effects of the saturated zones and of the seed radiation that the masers amplify can be neglected.

These fluctuations may have been detected in recent observations by Clegg \& Cordes of Galactic $\mathrm{H} \mathrm{II/OH}$ masers, providing a possible direct determination of the saturation intensity $J_{s}(=\Gamma / 2 B)$, an important input parameter for theoretical modeling. Long time-scale variations in the fluctuation amplitudes can provide information on phenomena such as passage of hydrodynamic waves in the maser region.
\end{abstract}

Subject headings: interstellar: molecules - masers - polarization

\section{INTRODUCTION}

Recent OH maser observations by Clegg \& Cordes (1991) show that the intensity amplitude in narrow frequency bands, much narrower than the thermal line width, exhibits fluctuations. The averages of the fluctuation amplitudes in various sources are typically $\sim 10 \%$, and the time scales range from a couple of minutes to an hour. If these time scales are interpreted as light travel times, the resulting dimensions are about two orders of magnitude smaller than those obtained from interferometry. Thus the masers flicker over dimensions much smaller than those resolved by observations that average the intensities over long times. Astronomical masers generally appear stable over time scales much longer than typical light crossing times for their entire lengths, which can be as long as days. Standard maser theory is derived under the assumption that the system is in steady state on these time scales, so this assumption appears justified for the mean intensities of most masers. The new observations indicate that fluctuations around the mean should be considered. This Letter attempts a first study of this phenomenon.

Because the system is so much longer than the wavelength, rays that move at slight angles do not interact directly. Similarly, the radiation bandwidth is sufficiently broad that different parts of it are uncorrelated, too. But the intensity of a ray propagating in a given direction at a given frequency is produced by coherent action along the entire ray path, as evidenced by the enormous brightness temperatures, and thus is susceptible to fluctuations in any part of the system. This is evident from the expression for the intensity at an arbitrary location $\ell$ :

$$
I_{v}(\ell)=I_{v}(l) \exp \tau_{v}(l, \ell)
$$

(e.g., Elitzur 1990). Here, $\tau_{v}(l, \ell)$ is the gain from the fiducial point $l$ along the ray path, an arbitrary point except for the weak restriction that the intensity of the seed radiation can be neglected there. If $\ell$ is chosen as the exit point, this expression shows that every point along the ray path can be considered the input source of the emergent radiation. The gain factor $\tau_{v}(l, \ell)$ for subsequent amplification is established by the overall structure of the whole source, including saturation effects, and thus can be considered stable on time scales comparable at least to light crossing times for the entire length. But the intensity $I_{v}(l)$ can be affected by local effects and thus may be subject to fluctuạtions on much shorter time scales, which will then be displayed by the emergent intensity. The problem is to identify the proper scales for typical times and amplitudes that characterize such fluctuations.

\section{AMPLIFICATION FLUCTUATIONS}

Maser radiation in a given frequency within the line width is controlled by its coherent interaction with particles in an inverted population whose time behavior is described by the familiar rate equations

$$
\begin{aligned}
& \frac{d n_{2 v}}{d t}=p_{2 v}-\Gamma n_{2 v}-B J_{v}\left(n_{2 v}-n_{1 v}\right) \\
& \frac{d n_{1 v}}{d t}=p_{1 v}-\Gamma n_{1 v}+B J_{v}\left(n_{2 v}-n_{1 v}\right),
\end{aligned}
$$

where all the notations are standard. These equations determine the (absolute value of the) absorption coefficient according to

$$
\frac{d \kappa_{v}}{d t}=\Gamma\left[\kappa_{0 v}-\kappa_{v}\left(1+\frac{J_{v}}{J_{s}}\right)\right],
$$

where $J_{s}=\Gamma / 2 B$ and $\kappa_{0 v} \propto\left(p_{2 v}-p_{1 v}\right) / \Gamma$. Standard theory is derived under the assumption of steady state, that is, $d / d t=0$. The expression inside the brackets then vanishes, providing the input relation for the radiative transfer equation. Strict steady state requires averaging over times much longer than particle interaction times. The standard theory thus provides the mean solution at every point inside the source, while the maser system fluctuates around this mean on short time scales. To study these fluctuations, consider a local deviation from the steady state mean. This can be described as a fluctuation $\delta$ in the solution to the mean absorption coefficient through

$$
\kappa_{v}=\frac{\kappa_{0 v}}{1+J_{v} / J_{s}}(1+\delta)
$$


From equation (3), the time behavior of $\delta$ is governed by

$$
\frac{d}{d t} \frac{1+\delta}{1+J_{v} / J_{s}}=-\Gamma \delta .
$$

Consider now an unsaturated maser, then $J_{v}<J_{s}$ and

$$
\frac{d \delta}{d t}=-\Gamma \delta
$$

The fluctuation decays with the rate $\Gamma$. The standard expression for the absorption coefficient can thus be considered the mean at every point; fluctuations around this mean generated at random times will decay on the time scale $\Gamma^{-1}$.

The radiation is propagating at the speed of light, and during one fluctuation period it covers the distance

$$
\lambda_{c}=c / \Gamma \text {, }
$$

which will be referred to as the radiation correlation length. Denote by $\frac{1}{2} \Delta(\lambda)$ average relative deviations from the mean of local level populations over the length scale $\lambda$. The absorption coefficient involves the difference of two level populations that fluctuate independently. Therefore, $\Delta \kappa_{0 v}(\lambda)$, the mean fluctuation amplitude of the absorption coefficient over the same length, is

$$
\Delta \kappa_{0 v}=\kappa_{0 v} \Delta(\lambda) .
$$

That is, $\kappa_{0 v}$ fluctuates over one correlation length in the range $\kappa_{0 v}\left[1 \pm \Delta\left(\lambda_{c}\right)\right]$. If $I_{0 v}$ is the long-term time average of the intensity at an arbitrary point in the maser, the mean intensity one correlation length later is $I_{v}=I_{0 v} \exp \left(\kappa_{0 v} \lambda_{c}\right)$, and it fluctuates between

$$
\begin{aligned}
I_{v} \pm \Delta I_{v} & =I_{0 v} \exp \left\{\kappa_{0 v} \lambda_{c}\left[1 \pm \Delta\left(\lambda_{c}\right)\right]\right\} \\
& =I_{v} \exp \left[ \pm \kappa_{0 v} \lambda_{c} \Delta\left(\lambda_{c}\right)\right] .
\end{aligned}
$$

And because each point in the maser provides the input for the rest of the ray on its way out, these fluctuations will be reflected in the observed intensity. Thus the amplification process introduces intensity fluctuations whose time scale is characterized by $\Gamma^{-1}$ and whose mean amplitude is

$$
\begin{aligned}
F_{a} \equiv \frac{\Delta I_{v}}{I_{v}}= & \frac{1}{2}\left\{\exp \left[\kappa_{0 v} \lambda_{c} \Delta\left(\lambda_{c}\right)\right]-\exp \left[-\kappa_{0 v} \lambda_{c} \Delta\left(\lambda_{c}\right)\right]\right\} \\
& \simeq \kappa_{0 v} \lambda_{c} \Delta\left(\lambda_{c}\right)
\end{aligned}
$$

where the last equality applies when $\kappa_{0 v} \lambda_{c} \Delta\left(\lambda_{c}\right)<1$.

The parameter $\kappa_{0 v} \lambda_{c}\left(=c \kappa_{0 v} / \Gamma\right)$ emerges as an important quantity in determining the amplitude scale of maser intensity fluctuations. When the maser loss process is collisiondominated, this parameter is independent of density and is determined exclusively by the efficiency of the inversion cycle. By example, for an $\mathrm{OH}$ relative abundance of $10^{-5}$ and a line width of $0.5 \mathrm{~km} \mathrm{~s}^{-1}$, the absorption coefficient of the main lines is $\sim 10^{-18} \eta N \mathrm{~cm}^{-1}$, where $\eta$ is the relative inversion and $N$ $\left(\mathrm{cm}^{-3}\right)$ is the overall density. If we assume that the loss rate is dominated by collisions across the maser transition, with a rate coefficient of $\sim 10^{-9} \mathrm{~cm}^{3} \mathrm{~s}^{-1}$, the noise parameter becomes $\kappa_{0 v} \lambda_{c} \sim 10 \eta$. This result provides a reasonable first-order estimate for other masers too.

The amplitude $\Delta\left(\lambda_{c}\right)$ represents an average over the length scale $\lambda_{c}$ since all scales $\lambda \leq \lambda_{c}$ are sampled by the radiation during the time $\Gamma^{-1}$. Different portions of the source could contribute differently to intensity fluctuations, depending on the local value of $\Delta\left(\lambda_{c}\right)$ and the local fluctuation spectrum, which remains undetermined in this study.

The analysis applies to saturated masers just the same. The radiation emanating in any direction is dominated by rays that passed through the unsaturated core; thus, the ray intensity fluctuates as described upon exit from the core and later on from the source. Large fluctuations could affect the core boundary. An increase in the intensity emerging from the core will move the core boundary in, increasing the saturated volume by the appropriate amount to accommodate the added intensity with the same pump rates. This effect can be understood in terms of background amplification, discussed in detail by Elitzur, McKee, \& Hollenbach (1991). Passage through the saturated zone produces faster fluctuations that sample only length scales shorter than $\lambda_{c}$. If $J_{v}$ is the mean angle-averaged intensity at a given point in the saturated zone, then $x \equiv$ $J_{v} / J_{s}>1$. The absorption coefficient is reduced to $k_{0} / x$, and equation (5) shows that the fluctuation decay rate increases by $x$, so the flicker times are shorter. The radiation correlation length is then $\lambda_{c} / x$ and

$$
F_{a}(x)=x^{-2} \kappa_{0 v} \lambda_{c} \Delta\left(\lambda_{c} / x\right) .
$$

The variation range of $x$ is from 1, in the unsaturated zone, to a maximum $x_{m}=J_{v}(\ell) / J_{s}$, where $J_{v}(\ell)$ is the exit angle-averaged intensity. By example, $x_{m}$ is probably $\sim 25$ for the strongest $\mathrm{H}_{2} \mathrm{O}$ masers in Galactic star-forming regions. The range $\Delta x=1$ corresponds to the length scale $1 / \kappa_{0 v}$, which is shorter than the core size, thus the corresponding contribution of the unsaturated core to the fluctuations at the length scale $\lambda_{c} / x$ will dominate. The saturated zone is less "noisy" and its contribution to the fluctuations can be neglected.

\section{SEED RADIATION AND POLARIZATION}

In addition to the fluctuations just described, the emergent intensity is expected to reflect fluctuations in the seed radiation that starts the buildup of the ray intensity. The nature of the seed radiation has not been closely scrutinized in the literature, and for an obvious reason: the saturated maser is a selfregulating system and the emergent intensity can be expressed exclusively in terms of $J_{s}, \kappa_{0 v}$, and geometrical factors, independent of the seed radiation intensity. However, the strongest rays are those that traveled the entire length of the source. The intensity generated in the small region before the seed intensity can be neglected is itself the input to the solution described by equation (1) and any fluctuations in its magnitude will propagate as described above. The length of this region is of order $1 / \kappa_{0 v}$.

The structure of astronomical masers is obtained by solving the radiative transfer equation

$$
\frac{d I_{v}}{d l}=\kappa_{v} I_{v}+\epsilon_{v},
$$

where the absorption coefficient is taken from the steady state solution to the level population equations (eq. [4] with $\delta=0$ ) and $\epsilon_{v}$ is the volume emission coefficient of maser radiation. This equation, as well as the level population equations, are derived from a hybrid approach wherein the radiation field is treated classically while the level populations are described by quantum mechanics. This approach does not yield the emission coefficient $\epsilon_{v}$, which has to be added as a phenomenological term. Since the maser is expected to amplify the radiation generated internally in spontaneous decays, the standard pro- 
cedure is to use the emission coefficient due to spontaneous emission, that is, $\epsilon_{v} \propto p_{2 v} / \Gamma$.

Although the intensity of the seed radiation that triggers the maser amplification process is due to spontaneous decays, the interpretation of $\epsilon_{v}$ as a standard thermodynamics emission coefficient runs into immediate difficulties. These can be simply recognized by considering a situation in which the pump rate $p_{1 v}$ is chosen equal to $p_{2 v}$ while all the other parameters are unchanged. The absorption coefficient then vanishes, so the maser process is turned off. But the emission coefficient remains the same, and the equation of radiative transfer shows that the intensity $I_{v}$ grows without bound and there is no scale to guage the error involved; the only local length scale that the steady state formalism defines is $1 / \kappa_{0 v}$, which diverges in this example. The problem origin can be traced by considering the nature of the emission coefficient in different situations. In the case of radiation produced by ordinary, noninverted populations, the emission coefficient describes the radiation that would be generated in particle decays if all induced processes were turned off; namely, radiation that can be completely decoupled from further interactions and thus can be called incoherent (with the level population distribution). In contrast, in the case of maser radiation the emission coefficient describes the volume production of coherent radiation, namely, radiation capable of interacting in complete coherence with the inverted population. Instead of thermodynamic considerations, the nature of the seed radiation is determined by the requirement of steady state equilibrium with the inverted level populations.

The constraints imposed by steady state equilibrium on the possible modes of coherent radiation become evident when the vector nature of the electric field is taken into account. The radiation field is then described by three independent intensities, corresponding to the three polarization states $p=-1,0$, +1 . These intensities are usually described by the four Stokes parameters $I, Q, U$, and $V$, constrained by the condition $Q^{2}+U^{2}+V^{2} \leq I^{2}$ to ensure that the radiation field they describe is comprised of transverse waves only. Alternatively, three of the Stokes parameters can be replaced by linear combinations $I^{p}$ corresponding to polarizations defined with respect to a frame aligned with the particle quantization axis (Elitzur 1991). These intensities therefore couple directly to $\Delta m=p$ transitions between magnetic sublevels, and the three corresponding absorption coefficients obey

$$
\frac{d \kappa_{v}^{p}}{d t}=\Gamma\left[\kappa_{0 v}^{p}-\kappa_{v}^{p}\left(1+\frac{J_{v}^{p}}{J_{s}}\right)\right],
$$

where $J^{p}=\int I^{p} d \Omega / 4 \pi$. This result applies to all spins and is derived in complete analogy to equation (3) under the assumption that the magnetic sublevels are degenerate and share the same decay rate $\Gamma$. Consider now the situation when the pump rates are $m$-independent so that all three absorption coefficient $\kappa_{0 v}^{p}$ are equal. For simplicity, consider a linear maser (so that $J=I$ ) inclined at an angle $\vartheta$ to the quantization axis, sufficiently small that amplification can be neglected. The steady state solutions to equations (13) are established when all three intensities $I^{p}$ are equal to each other and independent of $\vartheta$. As shown in Elitzur (1991), this is the polarization solution of Goldreich, Keely, \& Kwan (1973). The establishment of steady state equilibrium involves the selection of the proper modes from all the waves in the radiation field. The phase differences between the different components of the selected waves are in accordance with the relations dictated by the pump rates, namely, they are in the proper polarization states. If the source is subject to an overall quantization axis, as is the case when it is permeated by an aligned magnetic field, the coherent radiation will be polarized. In the absence of such preferred direction, averaging over orientations of the quantization axis removes the polarization.

The full implications of steady state equilibrium will be discussed separately in a forthcoming publication. The relevant point for the present discussion is that the equilibrium between the seed radiation and the maser particles is established over the time scale $\Gamma^{-1}$, so this is the fluctuation time scale for the seed radiation in a weak maser. The corresponding steady state length scale is $\lambda_{c}$ and the volume emission coefficient of maser radiation is obtained from volume averages over dimensions larger than $\lambda_{c}$. As before, the relative mean deviation from steady state of level populations over the distance $\lambda_{c}$ is $\frac{1}{2} \Delta\left(\lambda_{c}\right)$, and the source function $S$, the intensity scale of the seed radiation, involves two levels that fluctuate independently. Therefore, the fluctuation amplitude of the seed radiation intensity in an unsaturated maser is

$$
F_{s} \equiv \frac{\Delta S}{S}=\Delta\left(\lambda_{c}\right)
$$

Comparing with equation (10), the strongest fluctuations will be contributed by the seed radiation whenever $\kappa_{0 v} \lambda_{c}<1$ and by the amplification process in the reverse situation. Over long time intervals, the ratio of the number of fluctuations contributed by each effect is $\kappa_{0 v} \ell-1$, the length ratio of the regions where the two processes occur.

In the case of a saturated maser, the seed for the radiation emanating from one end is produced in the far end, which is permeated by the saturated radiation that travels in the opposite direction. Fluctuations decay much more rapidly, by the factor $x_{m}$, and $F_{s}=\Delta\left(\lambda_{c} / x_{m}\right)$. Thus the seed radiation can be considered quiet on all scales of interest, and fluctuations in saturated masers are dominated by the passage through the unsaturated core.

\section{DISCUSSION}

The analysis here describes only an intensity fluctuation due to a single disturbance along the ray path. In reality, the ray may sample various locations where the level populations happen to deviate from their local steady state mean. This will lead to intensity fluctuations that sometimes add together and at other times subtract from each other, and will determine the statistics and spectrum of maser intensity fluctuations. The input for a detailed analysis of these properties is the single "scattering" event described here, which shows that the parameter $\kappa_{0 v} \lambda_{c}$ provides a basic unit of maser noise.

Two other fundamental complications arise from the practical limitations of observations: First, intensity is measured only when an individual maser feature is spatially resolved, while single-dish observations measure flux - the product of the intensity and the maser beaming angle, roughly the solid angle occupied by the core at the exit point. A more complete analysis will thus have to include also modeling of the transverse dimensions of the fluctuations in the unsaturated core. This introduces a certain degree of uncertainty and a dependence on the specific geometry; it may be noted, however, that although the core size is larger than the correlation length $\lambda_{c}$, the ratio of the two is generally only a few. Second, observations always sample a finite bandwidth rather than a sharp 
frequency, and thus average over many units of the fundamental bandwidth $\Gamma$. A realistic modeling of the amplitude $\Delta\left(\lambda_{c}\right)$, which must take into account such effects as Alfvén waves, etc., will need to consider the time-sampling method used in a given ' experiment.

As before, these two effects only enter in the analysis of the amplitudes (magnitude, occurrence frequency of large fluctuations, etc.), not the time scales; while a detailed modeling of amplitudes involves unavoidable complications, the analysis of time scales appears reasonably simple and free of assumptions about detailed structure or dynamics. Fluctuation measurements can thus determine directly the saturation intensity $J_{s}$, an important input parameter for theoretical modeling. A trend of long-term variations in the fluctuation amplitudes can indicate the passage of a hydrodynamic wave, for example. The study of maser fluctuations provides potential probes of very detailed properties of the host environments.

If the fluctuations described here are those observed by Clegg \& Cordes, loss rates for $\mathrm{OH}$ masers are $\sim 10^{-2}$ to $10^{-3}$ $\mathrm{s}^{-1}$ and the coherence length is $\lambda_{c} \sim 10^{12}-10^{13} \mathrm{~cm}$. If losses are dominated by collisions, the densities are $\sim 10^{6}-10^{7} \mathrm{~cm}^{-3}$, in good agreement with independent estimates. Furthermore, the noise parameter $\kappa_{0 v} \lambda_{c}$ is independent of density and its value is $\sim 10 \eta$, where $\eta$ is the inversion efficiency. The observations indicate that typical fluctuation amplitudes are $\sim 10 \%$. From equation (10), a single fluctuation along the ray path contributes an amplitude of $10 \eta \Delta\left(\lambda_{c}\right)$ and could explain the observations if both $\eta$ and $\Delta\left(\lambda_{c}\right)$ were of order $10 \%$, for example. The absence of fluctuations in the one late-type star Clegg and Cordes observed can be attributed to either lower inversion efficiency, or smaller $\Delta\left(\lambda_{c}\right)$, or a combination of both. Since late-type stars provide a less active environment than $\mathrm{H}$ II/OH regions, a smaller $\Delta\left(\lambda_{c}\right)$ is probably to be expected. The simple theory presented here appears to be in accord with $\mathrm{OH}$ observations for reasonable parameters. Other masers can be expected to display similar fluctuations, though on different time scales. Loss rates for $\mathrm{H}_{2} \mathrm{O}$ and $\mathrm{SiO}$ masers are typically estimated to be $\sim 1 \mathrm{~s}^{-1}$, so fluctuation time scales are only seconds.

In addition to the intensity, the polarization can be even more strongly affected by fluctuations around steady state along the ray path. Independent fluctuations of the different intensities $I^{p}$ introduce an effect similar to random Faraday rotation by independent amounts for clockwise and counterclockwise rotations. Indeed, the two circular polarization states generally display a different behavior in the OH observations (Clegg 1990). In the case of linearly polarized radiation, this effect will tend to transfer the polarization of independent waves from $Q$ to $U$. Since $U$ vanishes when averaged over all modes, the polarization of the emerging radiation could be reduced from its strict steady state value (short-term fluctuations around the mean aggregate to produce an effect comparable to the mean itself). It thus seems that the study of maser polarizations cannot be divorced from the study of fluctuations along the ray path.

Finally, the stability of steady state maser solutions against intensity perturbations can be studied using the coupled set of equations (3) and (12). A detailed analysis is a difficult problem, which fortunately can be avoided. Masers are rather stable systems because any increase in the intensity reduces the absorption coefficient, thanks to the saturation effect, thus reducing the amplification and working in the opposite direction to the perturbation. The overall system readjusts in about one light-crossing time, and long-term fluctuations, on this time scale, should be minimal. There appears to be, however, one situation in which these fluctuations could become substantial. When the maser length increases, the core shrinks, and for sufficiently long dimensions, the core saturates (Goldreich \& Keeley 1972). In the steady state solution, the flux across the core vanishes, because rays that move in opposite directions have the same intensity, and $J_{v}$ is constant and larger than $J_{s}$ (see also Elitzur 1990). It is easy to show that the condition for core saturation is that the core size become equal to $\lambda_{c}$, the radiation correlation length; thus, incoming radiation fluctuates on core crossing times. Since both streams contribute equally to the location of the core, perturbations in one of the streams can move the core in the opposite direction, inducing oscillations. The overall system will again readjust on total light-crossing times, but during the readjustment period the maser can emit spectacular bursts of intensity. The reason is that portions of the core can become temporarily unsaturated, during which time there will be brief exponential amplification of radiation whose intensity is characterized by the saturation intensity $J_{s}$ rather than the source function $S$.

This conjectured behavior is strongly reminiscent of the two giant bursts observed in $\mathrm{H}_{2} \mathrm{O}$ maser emission, especially the one in Orion (Matveenko 1986; Garay, Moran, \& Haschick 1989). If this proposal proves correct, it may also have important implications for extragalactic $\mathrm{H}_{2} \mathrm{O}$ megamasers, where a higher concentration of maser sources could be expected to produce more cases of core saturation.

I am greatly indebted to M. M. Litvak and C. F. McKee for most insightful comments that were crucial for the formulation of the ideas presented here. I also thank A. W. Clegg for a lucid and informative discussion of the observations, and P. Goldreich for critical comments on the manuscript. This work was supported in part by NSF grant AST 87-16936.
Clegg, A. W. 1990, private communication

Clegg, A. W., \& Cordes, J. M. 1991, ApJ, in press

Elitzur, M. 1990, ApJ, 363, 628

. 1991, ApJ, 370, 407

Elitzur, M., McKee, C. F., \& Hollenbach, D. J. 1991, ApJ, 367, 333

\section{REFERENCES}

Garay, G., Moran, J. M., \& Haschick, A. D. 1989, ApJ, 338, 244

Goldreich, P., \& Keeley, D. A. 1972, ApJ, 174, 517

Goldreich, P., Keeley, D. A., \& Kwan, J. Y. 1973, ApJ, 179, 111

Matveenko, L. I. 1986, Soviet Astr., 30, 589 